\title{
A Wireless Intelligent Business Laundry Service System
}

\author{
Yujie Qiao, Can Zhang, Rui Li, Zhi Liu \\ Chengdu University of Technology, Chengdu, China \\ Email: 3032509054@qq.com
}

How to cite this paper: Qiao, Y.J., Zhang, C., Li, R. and Liu, Z. (2019) A Wireless Intelligent Business Laundry Service System. Journal of Computer and Communications, 7, 105-113. https://doi.org/10.4236/jcc.2019.77011

Received: June 3, 2019

Accepted: July 7, 2019

Published: July 10, 2019

Copyright () 2019 by author(s) and Scientific Research Publishing Inc. This work is licensed under the Creative Commons Attribution International License (CC BY 4.0).

http://creativecommons.org/licenses/by/4.0/

(c) (i) Open Access

\begin{abstract}
For the business washing machine industry, most are currently using traditional manual service (the way cost is high, inefficient, low degree of intelligence) of the status quo. The wireless intelligent business laundry service system based on ESP8266 communication module is developed, which has the Wechat payment function and realizes the automatic control service of the washing machine. Firstly, the laundry wireless terminal will send a laundry request to the laundry control center when the customer is ready to wash. Secondly, after receiving the laundry request, control center will send WeChat Public Accounts, idle washing machine number and charges, as well as laundry service time and other information back to the wireless terminal to generate payment QR code. And then the customer scans QR code, when the control center receives the information that payment is successful, the system will automatically control the washing machine to complete the laundry service work. After testing, the system has the advantages of accurate control, low energy consumption, strong stability, accurate data transmission, low cost, strong practicability, and is conducive to promoting the development of the business laundry industry.
\end{abstract}

\section{Keywords}

Wireless, Intelligent, Business Laundry Service, Esp8266

\section{Introduction}

The application of the Internet of things (IoT) technology in the business washing machine industry is conducive to saving manpower, material resources, cost saving and service efficiency [1]. According to the present situation analysis and research on the Chinese market of washing machine industry in 2016 forecast released from the China Industry Research website, due to the accelerated pace 
of the city, more and more people choose to wash in the laundry. Most of the laundry business employ the staff to charge and manual mode to control machines [2]. This mode of operation is primitive and it is not conducive to the development of business washing machine industry [3]. In public places such as schools and hospitals, more and more people choose to use washing machines. Traditional coin-operated washing machines are complicated. In addition, with the development of intelligent payment platform, users rarely bring their own change, so the traditional laundry service system has been unable to meet the needs of customers [4]. It is necessary now to improve business laundry service to meet the requirements of the development by means of improving the quality of science and technology.

This paper describes the design of a wireless intelligent business laundry service sytem based on network payment platform, through the washing control center and WeChat business platform information exchanging, the system can realize the customer laundry, from order, payment, laundry control and other service flow automatically. On the other hand, the payment system through online payment platform solves the problem that the coin-operated laundry system needs to provide small change process, the simple operation is conducive to promoting the development of washing machine industry.

\section{System Structure Design}

As shown in Figure 1, the wireless intelligent business laundry service system consists of WeChat merchant platform, laundry control center, wireless terminal for washing machine and WeChat client. When a customer needs laundry service, a service request is sent through the wireless terminal of the washing machine. When the laundry control center receives the service request, which will generate a unique order number and package the relevant information of WeChat public number, order ID, merchant number, payment information and the local time and sends to the wireless terminal of washing machine through UDP protocol [5] [6]. When the wireless terminal of the washing machine receives the

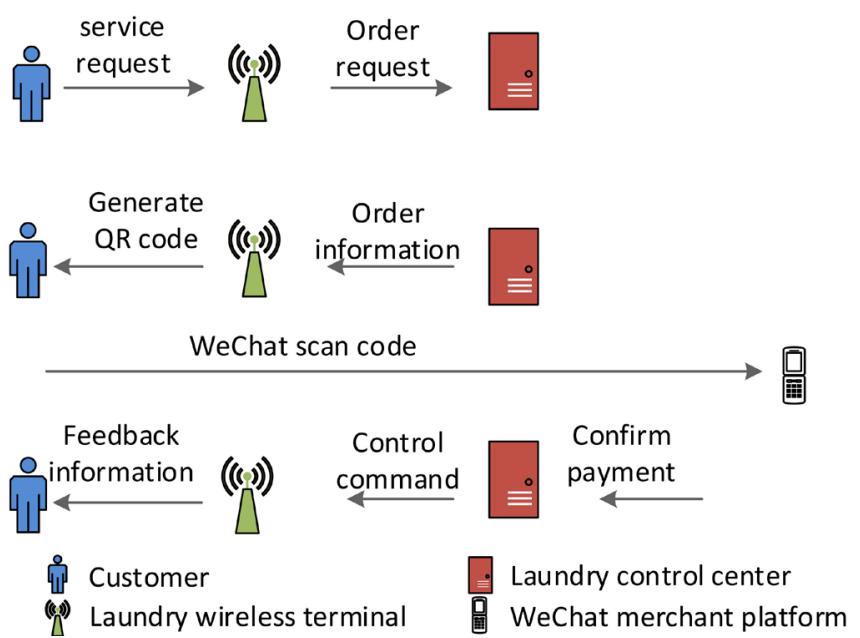

Figure 1. System structure diagram. 
above information, a random signature is generated according to the built-in algorithm, then generates an order QR code with local time and displays it. Customers can scan the code through the Mobile WeChat client, confirm the order and make payment after the successful payment, WeChat merchant platform will send the payment information to the laundry control center, the laundry control center generates the corresponding control commands according to the order price, and sends the commands to the wireless terminal of the washing machine, finally, the wireless terminal controls the washing machine operation according to the control commands, including laundry mode, washing time, etc. At the same time, the information is displayed on the touch screen and fed back to the customers.

The washing control center has the function of wireless transmission and network access, in this system, receiving the information of order and payment from WeChat merchant platform through the network, and then wireless transmission to the washing machine wireless terminal, control the washing machine work.

The washing machine wireless terminal is composed of MCU, wireless module, touch screen, control module and power module. The whole structure is shown in Figure 2, when an order is generated, the laundry control center sends the information of this order, such as WeChat Public Accounts, Spare washing machine number, fee scale, laundry service time and so on, when the laundry wireless terminal receives the information through the wireless module, transmits it to MCU, MCU generates 32 bytes random numbers according to the built-in algorithm, and then generates the signature through the MD5 code, and integrates the above information to generate $\mathrm{QR}$ code for payment of goods. After completing the above process, encrypting the corresponding response message by CRC16 then transferring to the laundry control center [7] [8], and waiting for the laundry control center to confirm the payment information. After the customer completing the payment, the laundry wireless terminal receives the payment confirmation information sent by the server, MCU analyzes the information and

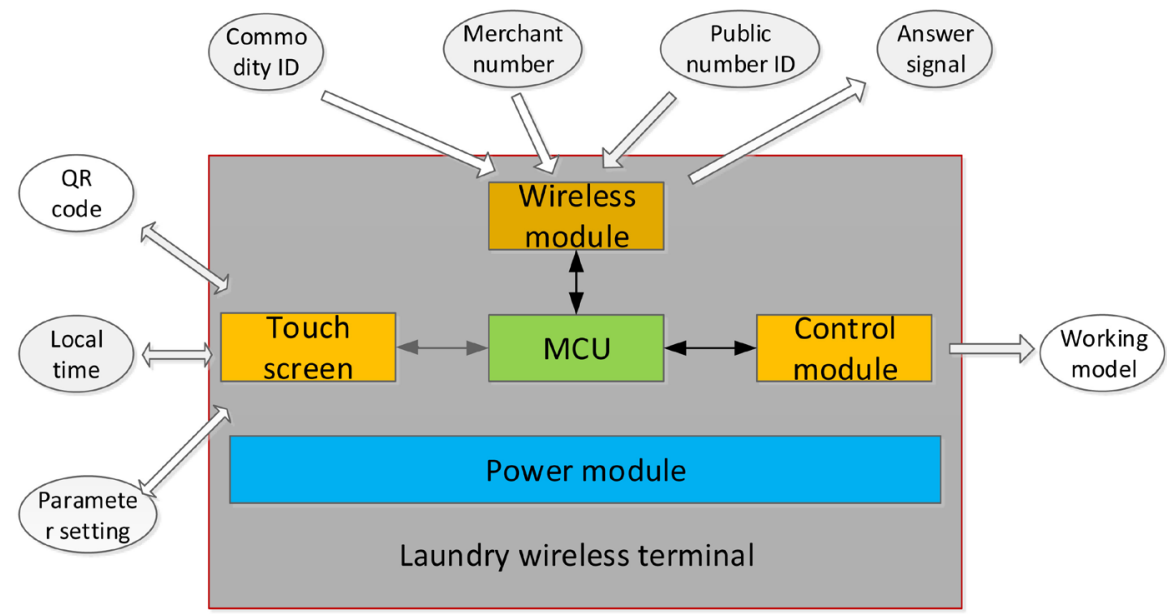

Figure 2. The structure diagram of washing machine control system. 
controls the washing machine through the control module, including washing mode, time and so on. At the same time, the information is sent back to the customer through the touch screen, and finally realizes autonomous laundry service.

Among them, MCU uses STM32F103RCT6 of STM32 company, this chip adopts the CortexTM-M3 kernel, has 256KFlash, 5 serial ports output, which could meet the need of display module, wireless communication and software debug. The wireless communication module adopts ESP8266 module, the module is built with TCP/IP protocol stack, and has UDP/TCP transmission modes. The selection of communication protocol and the design of logical structure of data packet are directly related to the stability and accuracy of communication, in this system, UDP communication is used between the ESP8266 [9] [10] module and the server, the data package format as shown in Table 1, using network order to send, that is, the high data bytes sent first, and low byte sent later.

There are 4 kinds of packet types, which consist of reservation $(0 \times 00)$, heartbeat $(0 \times 01)$, parameter request $(0 \times 02)$ and parameter settings $(0 \times 03)$.

Reservation: save the initial settings of the system.

Heartbeat: heartbeat packet is used to communicate with control center and wireless terminal washing laundry, and wireless terminal receives a heartbeat washing instructions every 5 minutes from washing control center and returns a response packet, if the washing control center fails to receive the response data 5 times, washing terminal ID will be uploaded and send the error.

Parameter request: the wireless terminal receives the parameter request data packet sent by the laundry control center, finishes the reading of the parameter type in the packet, and transmits to the laundry control center.

Parameter setting: the wireless terminal receives the parameter setting command sent by the laundry control center and executes the response instruction. In order to ensure the integrity and accuracy of data transmission process, CRC16 calibration is introduced.

The touch screen selects DC48270043C_03, which is a 4.3 inch ommercial configure serial port screen with a resolution of $480 * 272$, built-in CORTEX-M3 kernel + high-speed FPGA processor, and the storage space is 1 Gbit [11]. The designed control platform of washing machine is download through the RS232 serial port, includes login, server selection, authority management, network parameter display, washing machine working mode setting and so on, at the same time, short circuiting the Serial port screen mode selection port J5 to Select TTL

Table 1. Data packet format list.

\begin{tabular}{ccc}
\hline Type & Bytes & Notes \\
\hline Session ID & 2 & Session number, increase progressively \\
Command_Type & 1 & Command type \\
Data_Len & 2 & Command/answer data length \\
Data_Body & Data_Len & Command/response data \\
CRC16 & 2 & Current packet CRC16 checksum
\end{tabular}


Table 2. The instruction list display module.

\begin{tabular}{|c|c|c|c|c|}
\hline $\begin{array}{c}\text { Serial } \\
\text { number }\end{array}$ & Function & Instructions & Instruction parameter & Explain \\
\hline 1 & Handshake & $0 \times 04$ & $\mathrm{~N} / \mathrm{C}$ & $\begin{array}{l}\text { The screen receives the handshake command number and } \\
\text { returns } 55 \text { to the host to indicate the handshake is successful. } \\
\text { Return format: EE } 55 \text { FF FC FF FF. }\end{array}$ \\
\hline 2 & $\begin{array}{l}\text { Update network } \\
\text { data }\end{array}$ & $0 \times \mathrm{B} 1+0 \times 11$ & Screen_id + Control_id + Strings & $\begin{array}{l}\text { Enter text data for the specified text control: } \\
\text { Screen_id: The display interface ID of Network parameter. } \\
\text { Control_id: The display control ID of Network parameter. } \\
\text { Strings: Update network settings parameters. }\end{array}$ \\
\hline 3 & $\begin{array}{l}\text { Read setup } \\
\text { parameters }\end{array}$ & $0 \times \mathrm{B} 1+0 \times 10$ & Screen_id + Control_id + Strings & $\begin{array}{l}\text { Read parameters for user setting: } \\
\text { Screen_id: Setting the parameter display interface ID. } \\
\text { Control_id: Setting the parameter display control ID. } \\
\text { Strings: Update settings parameters. }\end{array}$ \\
\hline 4 & $\begin{array}{l}\text { Read user function } \\
\text { operations }\end{array}$ & $0 \times \mathrm{B} 1+0 \times 00$ & Screen_id & $\begin{array}{l}\text { Switch to the interface of user selected function: } \\
\text { Screen_id: Function interface ID }\end{array}$ \\
\hline$\cdots$ & $\cdots$ & $\cdots$ & $\cdots$ & $\cdots$ \\
\hline
\end{tabular}

level, the exchange of information with the STM32 processor is implemented by an external STM32F103RCT6 Usart2, and Realize the function of real-time display, Some instructions are shown in Table 2.

\section{The Design of Software System}

The program of wireless intelligent business laundry service system has function of the network connection between the washing machine control system and the merchant server, module initialization, interrupt monitoring and processing, real-time error reporting, privilege management and so on, the specific working process is shown in Figure 3.

1) ESP8266 module initialization, Touch screen initialization, Monitoring port initialization, GPIO initialization.

2) Wait for ESP8266 to connect to the network.

3) Check the status of network connection.

Success: proceed to the next heartbeat program.

Failure: returning 2), continue to connect, if multiple connections failed, the error was reported.

4) End heartbeat packet.

5) Detect whether there is an interruption.

No: $n$ plus 1.

Yes: $<1>$ Serial port interrupt $\rightarrow$ Data verification $\rightarrow$ Serial port operation program.

$<2>$ SP8266 interrupt $\rightarrow$ RC check $\rightarrow$ Execute the ESP8266 interrupt program: Payment status, serial screen display, washing machine working mode, etc.

6) $n$ value check.

$\mathrm{n}>\mathrm{c}$ (error tolerance), system error, alarm, inform staff to check the wrong equipment.

$\mathrm{n}<\mathrm{c}$, the procedure repeat step 5). 


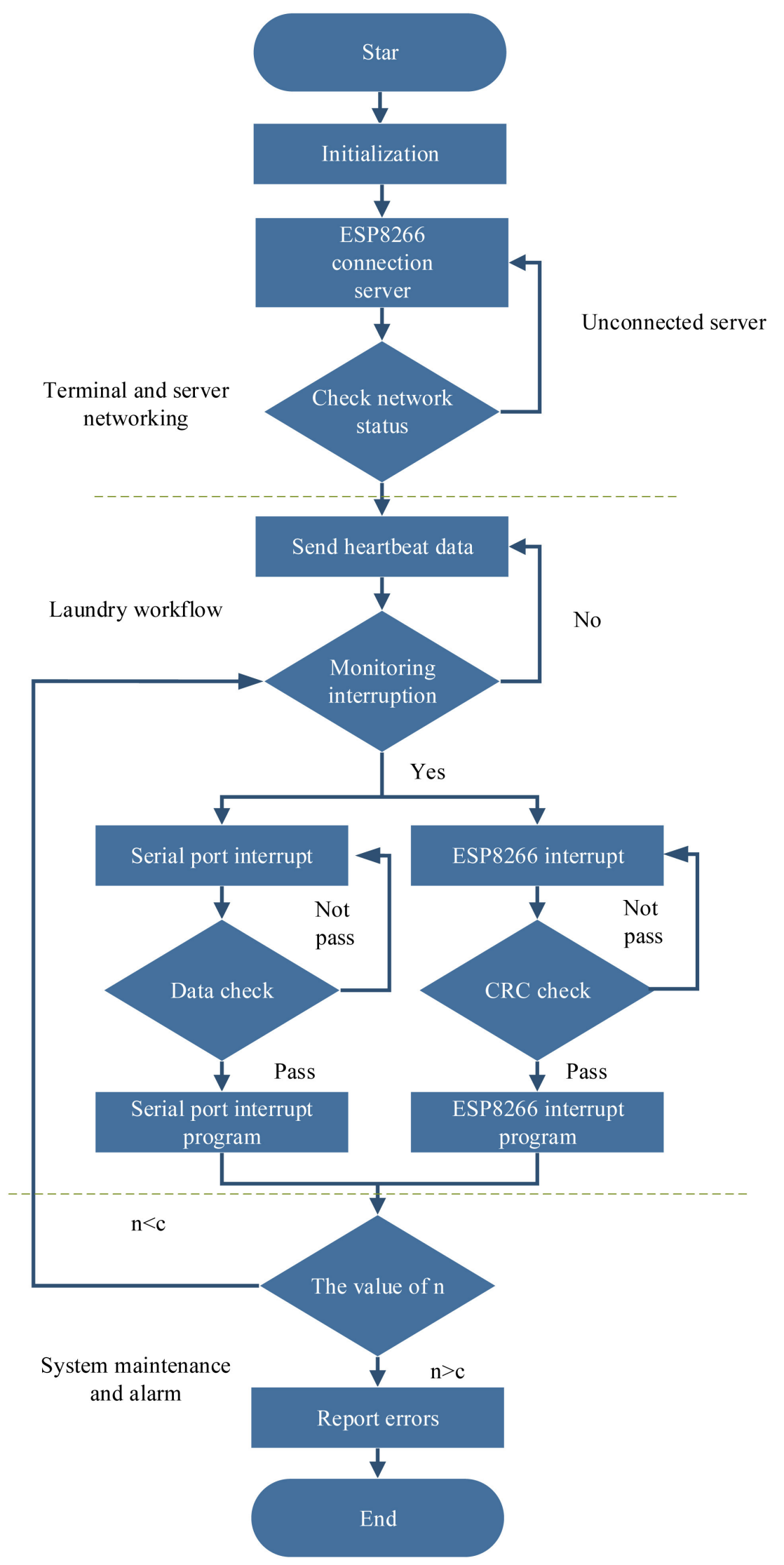

Figure 3. System program flow chart. 


\section{Test of Application}

The wireless intelligent business laundry service system has been successfully applied in a washing machine shop in Chengdu, the washing machine platform used in the washing machine is shown in Figure 4. The interface information is shown in Figure 5, including QR code scanning, Settings, Laundry and User information. Parameter Settings are shown in Figure 6, including wireless network name, password, server and other information. When the user prepares to wash clothes, the customer and the clerk send the laundry request to the washing machine through the touch screen, and then the control center sends the WeChat account number, laundry time and other information back to the wireless terminal to generate the payment QR code is shown in Figure 7. After the user makes the payment through the QR code, The washing machine starts to work. The laundry terminal and laundry service control center are shown in Figure 8, the display shows that the order was paid successfully, the laundry program has been started, and the washing machine starts to work, and the data returned is correct, which proves that the control system can be used normally.

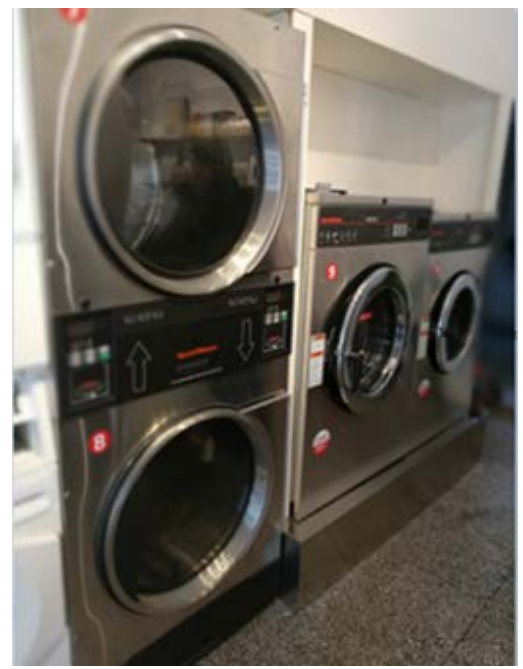

Figure 4. Washing machine platform.

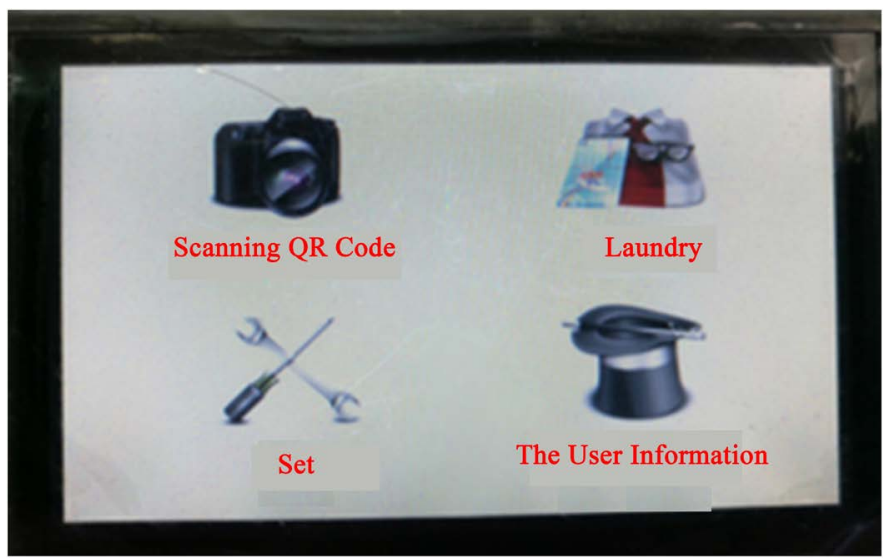

Figure 5. Interface information. 


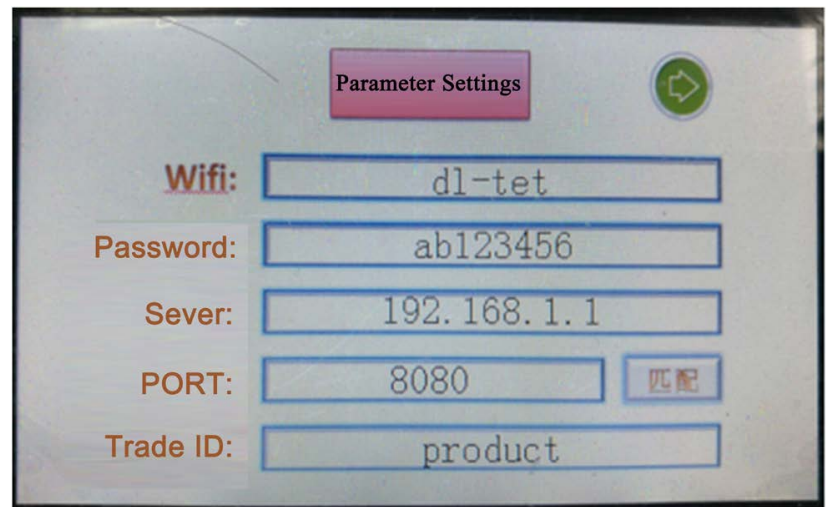

Figure 6. Parameter Settings.

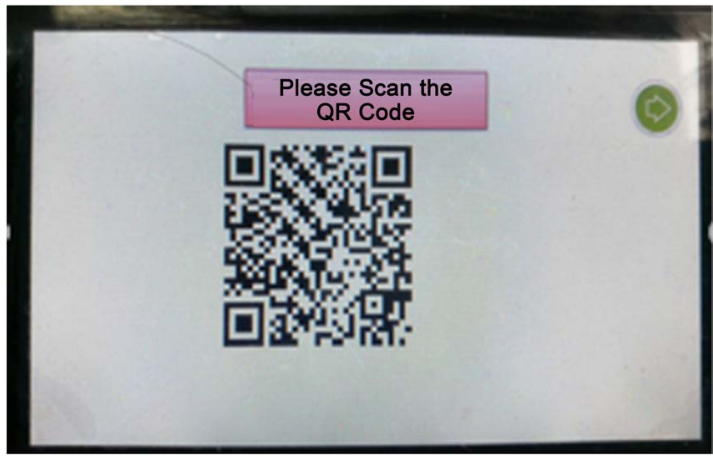

Figure 7. Generate QR code.

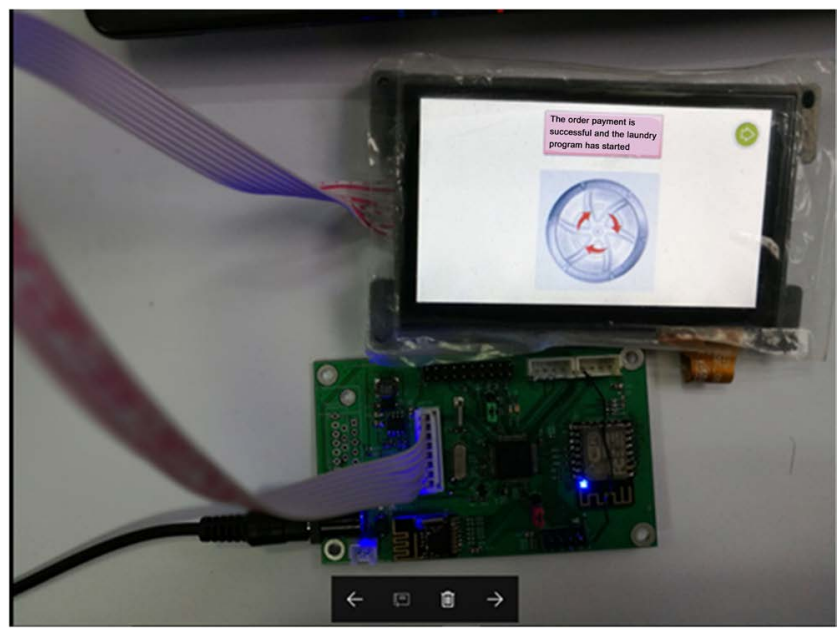

Figure 8. The laundry terminal.

\section{Conclusion}

This paper designs a wireless intelligent business laundry service system based on ESP8266 wireless communication module and WeChat payment platform, which can realize customer laundry, payment and laundry control at the same time. Through the application test of the laundry, the system has the characteristics of high intelligence, high efficiency and high stability, which is beneficial to the intelligence of the business laundry industry. 


\section{Acknowledgements}

This work was supported by Sichuan Science and Technology Program (2018TJPT0008, 2019YFG0430) and key project number of department of education (17ZA0023).

\section{Conflicts of Interest}

The authors declare no conflicts of interest regarding the publication of this paper.

\section{References}

[1] Gu, J. and Du, Q. (2016) The Design of Intelligent Washing Machine Controller Based on FPGA. Fifth International Conference on Instrumentation and Measurement, Computer, Communication and Control, Qinhuangdao, 18-20 September 2015, 1529-1532. https://doi.org/10.1109/IMCCC.2015.324

[2] Ravichandran, S. (2016) Colour Discharge Alarming System in Washing Machine Using Internet of Things. International Journal of Control Theory \& Applications, 9, 147-150.

[3] Yu, J.S. (2005) Design of Intelligent Washer Based on Fuzzy Control. Journal of Guangdong Polytechnic Normal University, No. 6, 41-43.

[4] Masood, R.F. (2017) Application of Fuzzy Logic in Design of Smart Washing Machine. Computer Science, arXiv: 1701.01654.

[5] Wang, G.J., Wang, Z.X., Xu, H.D. and Zhang, X.G. (2010) The Application of Fuzzy-Neural Network Based on HGA in the Washing Machine Control System. 2010 International Conference on Intelligent Computation Technology and Automation, Changsha, 11-12 May 2010, 702-705. https://doi.org/10.1109/ICICTA.2010.49

[6] Kurniawan, W., Ichsan, M.H.H., Akbar, S.R., et al. (2017) Lightweight UDP Pervasive Protocol in Smart Home Environment Based on Labview. IOP Conference Series: Materials Science and Engineering, 190, Article ID: 012009. https://doi.org/10.1088/1757-899X/190/1/012009

[7] Zhang, Y.X. (2007) Intelligent Online Dynamic detect and Data Processing System for Electric Machine of Washing-Machine. Control \& Automation, 23, 104-105.

[8] Hong, S. (2018) Washing Machine and Control Method Thereof.

[9] Azmi, N., Sudin, S., Kamarudin, L.M., et al. (2018) Design and Development of Multi-Transceiver Lorafi Board Consisting LoRa and ESP8266-Wifi Communication Module. IOP Conference Series: Materials Science and Engineering, 318, Article ID: 012051. https://doi.org/10.1088/1757-899X/318/1/012051

[10] Marques, G. and Pitarma, R. (2016) An Indoor Monitoring System for Ambient Assisted Living Based on Internet of Things Architecture. International Journal of Environmental Research and Public Health, 13, 1152. https://doi.org/10.3390/ijerph13111152

[11] Ning, H.-S. and Xu, Q.-Y. (2010) Research on Global Internet of Things' Developments and It's Lonstruction in China. Acta Electronica Sinica, 38, 2590-2599. 